\title{
Thermal Resistance Modeling for the Electrothermal Layout of High-Power RF Transistors
}

Peter H. Aaen, John Wood, Quan Li, and Eddie Mares

Freescale Semiconductor Inc., RF Division, Tempe, AZ, USA

\begin{abstract}
This paper demonstrates a practical approach to developing a geometrically scalable thermal resistance model to optimize layout for improved electrical performance of highpower RF transistors. The model is developed using finite element-based simulations, which show very good agreement with measured results. The proposed modeling methodology precomputes simulations over all possible layout considerations and the individual elements of the thermal resistance matrices are automatically approximated by thin-plate splines. This approach produces a model for use within a circuit simulator with virtually no overhead. We are able to scale the model up to $60 \mathrm{~mm}$ with less than than $2 \%$ error in the maximum predicted temperature rise.
\end{abstract}

\section{INTRODUCTION}

Under large-signal and high output power operation, RF transistors generate significant heat flux and the effects of high temperature operation on the electrical characteristics of the transistor must be carefully considered and controlled. During the design of the transistor the relationship between the geometrical layout and the temperature distribution across the transistor is usually studied empirically, and optimized for best electrical performance.

In the design of the semiconductor die, the number of fingers, spacing between fingers, and the unit-gate width can all be changed. These features are indicated in the photograph and illustration shown in Fig. 1. In general, the greater the total gate width within a package, the higher the output power of the transistor. The frequency of operation usually limits the maximum unit-gate width, and the device engineer is left to select the number of fingers and the pitch.

The dimensions of industry standard packages restrict the size of the silicon die, and hence the total silicon area. To accommodate more gate width within the package, the finger pitch must be reduced. For the same power dissipation, packing more power into a smaller area increases the operating temperature of the transistor to the detriment of the electrical performance. The thermal and electrical design considerations are coupled and a compromise must be reached. Many techniques have been devised to simulate coupled electrothermal problems ranging from the development of numerical techniques, to simulating the fully-coupled governing electrical and thermal equations [1], with several commerciallyavailable codes [2], [3]. These techniques are robust and can readily solve coupled-physics problems. Unfortunately, due to the large computational overhead of solving the coupled nonlinear differential equations numerically, these techniques are limited in the size of the problem they can address, and
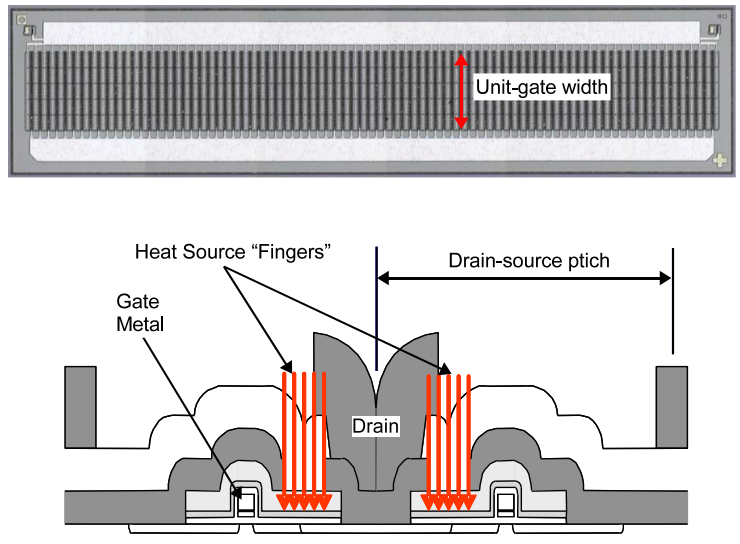

Figure 1. A photograph of a 100-mm LDMOS transistor indicating the unit-gate width and an illustration of the device cross section indicating the location of the heat source located between the gate and drain of the transistor.

they are not readily adaptable for use within the circuit-theory based simulator, where the RF transistor is designed.

For the design and simulation of RF power transistors, it is more appropriate to combine thermal and electrical transistor models in a compact form within the circuit simulator. The thermal models can be implemented directly into the circuit simulator [4], or imported into the circuit simulator using statespace representations [5], or through the use of thermal resistance matrices [6]. During a circuit-theory based simulation, the power dissipated by each transistor is computed and passed to the thermal model and the temperature rise is computed. The temperature is then passed to the electrothermal nonlinear transistor model [7] and a new dissipated power is computed and passed again to the thermal model. This process continues until convergence is reached. With the exception of Batty, who implements a complex fully analytical solution [4], little attention has been directed towards the generation and rapid simulation, within circuit-theory based simulators, of thermal models with large numbers of thermal ports to accommodate each transistor finger.

In this paper we present a practical thermal modeling methodology that can be used in conjunction with nonlinear transistor models for an electrothermal layout process. We demonstrate how this technique is used to compute rapidly the thermal resistance matrices of transistors with very large total gate widths, for example, 100-200 gate fingers, requiring 100-200 thermal ports. We demonstrate the model and its applicability using a finite-element based thermal simulator, ANSYS ${ }^{\mathrm{TM}}$. This technique is compatible with the majority 
of the aforementioned methods. As will be shown, we focus on an examination of the repetitive geometry of the transistor, and by careful application of linear superposition we reduce the number of simulations required to construct the thermal impedance matrix. The design space is sampled over possible die layouts, thermal simulations are performed, and we generate a mathematical description using function approximation techniques. Ultimately we arrive at a model that simulates almost instantaneously, but with the accuracy of the finite-element based simulations. This model can then be used to optimize the transistor layout for a given electrical application, in a computer-based design environment, rather than by experimental measurement of a matrix of different device structures. This saves both time and the expense of device fabrication and characterization in the design process.

\section{Measurements \& Finite-Element Simulation}

Measurements of the temperatures across the surface of the die are performed using infra-red (IR) microscopy. The IR microscope is integrated with an RF test-bench which allows temperature distributions to be measured under realistic termination impedances and signal excitations. Since silicon, is translucent to the infrared portion of the spectrum a coating is applied that ensures a constant emissivity surface while only impacting the RF performance minimally [8].

We are interested not only in the temperature of the transistor, but also in its thermal resistance. To compute this we need to know the maximum temperature on the die, the temperature at the thermal reference plane, and the amount of dissipated power. The constant emissivity coating is applied to the transistor, and the maximum temperature on the die is found using the IR microscope. A thermocouple is placed in contact with the back of the package and this temperature is captured. The dissipated power is computed as the product of the voltage and current at the drain (under DC operation), and $R_{t h}$ is computed by

$$
R_{t h}=\frac{\Delta T}{P_{d i s}}
$$

where $\Delta T$ is the temperature rise, and $P_{d i s}$ is the dissipated power (or incident heat-flux). Details of the measurement and calibration process are described in [9], [10].

To demonstrate the correlation between our measurements and simulations we biased an LDMOS transistor under DC conditions to obtain $4.8 \mathrm{~W}$ of dissipated power. The transistor has a total gate width of $4.8 \mathrm{~mm}, 600 \mu \mathrm{m}$ unit-gate width, $30 \mu \mathrm{m}$ source-drain pitch, and is built on an approximately 80 $\mu \mathrm{m}$ thick silicon substrate, and is mounted on top of $\mathrm{a} \approx 730$ $\mu \mathrm{m}$ thick copper carrier. The image in Fig. 2 is taken using a QFI InfraScope II $^{\mathrm{TM}}$ system outfitted with a $15 \times$ magnification lens resulting in a spot size of $1.6 \mu \mathrm{m}$. The graded temperature profiles both across and transverse to the direction of the fingers can be seen. Simulations of the same device were performed using the finite-element based software, ANSYS, and plots of the temperature profiles, as illustrated in Fig. 2 are shown in Fig. 3.

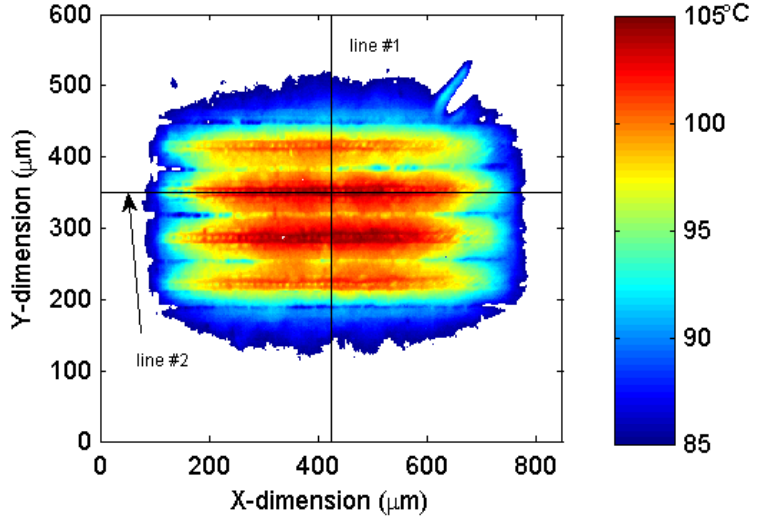

Figure 2. A plot of the temperature distribution across the surface of a LDMOS transistor under DC operation, $4.8 \mathrm{~W}$ dissipated power. The numbered lines indicates the location of the temperature profiles for Fig. 3.

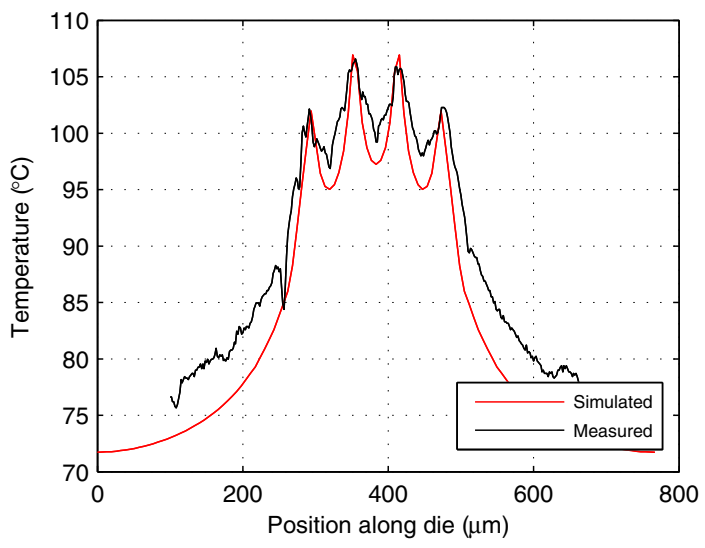

Figure 3. Measured and simulated temperature plots along line \#1 as shown in Fig. 2.

Heat sources for the LDMOS transistor are assumed to be between the gate and drain metal contacts as indicated in Fig. 1. Although we neglected the heat-spreading effects of the metallization on the die, as well as convective and radiative cooling, the results compare very well. With this good correlation between measurement and simulation, the finiteelement simulator can be used with confidence to provide data for model generation.

\section{Thermal Matrix \& Model Development}

The thermal resistance matrix of the transistor is obtained from simulation, by turning on an incident heat-flux at each finger and then examining the temperature over the whole die, and measuring the temperature at all other fingers. We proceed by exciting each finger in order, until the full thermal resistance matrix is obtained [6]. The temperature for a given finger is defined by averaging the temperature by integration over the finger area. Since the material properties change with temperature we are careful to perform these single source simulations at temperatures close to those expected in the solution [11]. Later we demonstrate how we can substantially reduce the number of simulations to obtain the thermalimpedance matrix based on geometrical considerations. 


\section{A. Scalability}

The size and complexity of the structures that can be simulated are determined by the efficiency of the numerical algorithms and the computer processing power that is available. With advances in both these areas, structures that were not practical to simulate a decade ago can now be solved routinely.

This rapid simulation time means that it is possible to sample the entire design space for the transistor, that is, the number of gate fingers, unit gate finger width, and source-drain pitch, in a reasonable time. Using function approximation techniques, we developed a mathematical model that can compute the thermal resistance matrix for any combination of these design variables. The parameterized description language available in ANSYS (APDL), was used to create a parameterized geometry to cover the design space. We developed a suite of Matlab ${ }^{\mathrm{TM}}$ functions to control the operation of ANSYS, to perform post-processing of the simulation results, and to generate the thermal model functions.

We used multivariate splines, employing simple bivariate spline construction that is provided in the Matlab Spline Toolbox [12]: the thin-plate smoothing spline.

$$
f(x)=\sum_{j=1}^{n-3} a_{j} \Sigma\left(x-c_{i}\right)+\text { linearterms }
$$

in which the $a_{j}$ are coefficients, the $c_{j}$ are centers in the data space, and $\Sigma(x)=\left|x^{2}\right| \log \left(x^{2}\right)$ is the radially-symmetric function. In this expression the summation runs to $n-3$, with the remaining terms being accommodated by the linear terms in the expression. The thin-plate spline minimizes the following expression over the function $f$ :

$p \sum_{i=1}^{n-3}\left|y_{i}-f c_{i}^{2}\right|+(1-p) \int\left(\left|D_{1} D_{1} f\right|^{2}+2\left|D_{1} D_{2} f\right|^{2}+\left|D_{2} D_{2} f\right|^{2}\right)$

In this model we approximated the change in all of the terms of the thermal impedance matrix. Through software control over the ANSYS simulation and the function approximation, the modeling process is fully automated.

To demonstrate the process we sampled uniformly over a source-drain pitch range of 20 to $60 \mu \mathrm{m}$ and unit-gate widths from 100 to $1000 \mu \mathrm{m}$, for a 16 finger transistor. Seventy ANSYS simulations were performed and the results tabulated for function approximation. From this data we generated a model of the thermal impedance matrix.

In Fig. 4, the first element of the thermal impedance matrix, $R_{t h 11}$, obtained from finite element simulations is shown and it is a very smooth function of source-drain pitch and unit-gate width. Slices of this surface for constant pitch and constant unit-gate width are shown to the right of the figure, where the results of the finite-element simulations are indicated with a plus symbol and the corresponding results from the model are indicated by circles. The largest difference over all points is less than $1 \%$ percent. Although not shown, similar results were obtained for all of the other elements of the thermal resistance matrix.

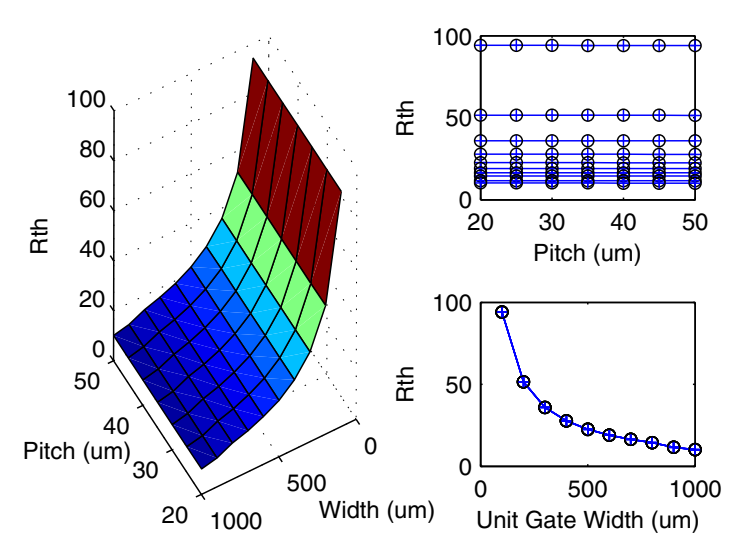

Figure 4. Simulation results of the $R_{t h 11}$ term and the constant slices as a function of gate-width and source-drain pitch are shown. The plus symbol are points obtained through finite-element simulations and the circle are the corresponding points computed from the model.

\section{B. Symmetry \& Geometry Considerations}

The number of simulations required to obtain the full $\mathbf{R}_{t h}$ matrix can be reduced by examining the repetitiveness of the transistor layout. The device is symmetrical so we only need to determine the thermal resistance terms in the main and upper diagonals. Furthermore, many of the terms in the upper diagonal are identical. In fact, for a series of equidistant heatsources only the top row of the thermal matrix needs to be computed. All nearest neighbour, next nearest neighbour, and subsequent neighbour interactions, as well as the self thermal resistance are included within the first row of the matrix.

For more complicated layouts, such as the one in Fig. 1, where two heat-sources are very close to each drain, we need to simulate the first row of the matrix plus additional terms in the second row to obtain the thermal resistance matrix for the entire structure.

\section{Scaling with number of fingers}

The temperature increase caused by a single heat-source drops quickly as a function of the distance from that heatsource. This manifests itself within the thermal impedance matrix by terms far away from the main diagonal tending to zero. Thus after some distance away from the heat source, its contribution is minimal and the matrix element can be replaced with a zero. For a given layout, this matrix can be used to generate the thermal impedance matrix for any sized transistor [13].

To determine this elementary $\mathrm{R}_{t h}$-matrix, we simulated a transistor with a large number of ports and we compute the peak temperature when all ports are excited from a uniform heat flux, mimicking the operation of the transistor. We included systematically more terms of this matrix, starting with the main diagonal only, then including successive offdiagonals, with all other terms set to zero. At each step we computed the peak temperature of the transistor and compare this value with the value obtained using all elements of the matrix. To obtain less than $1 \%$ error for a transistor having a finger length of $600 \mu \mathrm{m}$ and a source drain pitch spacing of 30 $\mu \mathrm{m}, 63$ diagonals need to be included (main diagonal, and 31 


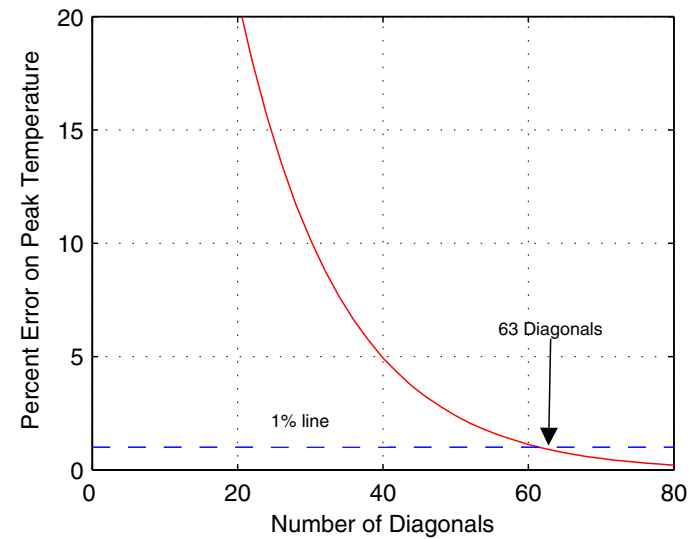

Figure 5. Plot of the percentage error between the maximum temperature on the transistor when the entire thermal resistance matrix is used vs matrices constructed that contain an increasing number of off-diagonal elements.

diagonals above and below the main), as shown in Fig. 5. With these results, we can determine a thermal resistance matrix for any number of fingers. Note that there is more inter-finger coupling for transistors with less spacing between heat sources, so when applying this technique to determine an elementary $\mathrm{R}_{t h}$-matrix over a large design space, the analysis should proceed on the layout with the highest inter-finger coupling.

\section{REsults}

To demonstrate the scalability and accuracy of the modeling methodology compared with finite-element simulations, we selected a 32-finger transistor as the base model. Using the matrix selection techniques described in the previous section, the thermal resistance matrices for 16, 32, 64, and 98 finger transistors were developed. For transistors with less fingers than the base model, the entire $\mathbf{R}_{t h}$ matrix is filled. While for transistors with more fingers than the base model, the $\mathrm{R}_{t h}{ }^{-}$ matrix is a banded around the diagonal with elements towards the upper and lower corners of the matrix set to zero. These zeros correspond to mutual resistance terms that are negligible.

A comparison of the model was made using the same incident heat-flux and the resulting temperature profiles across the die was computed. As shown in Fig. 6 there is almost no discernible error for scaling to lower gate peripheries, while the error increases only slightly for larger periphery transistors. The error results from dropped diagonals in the $\mathrm{R}_{t h}$ matrix, whose values cannot be obtained from the base model. The maximum error on the thermal profile is less than $2 \%$ or $0.6^{\circ} \mathrm{C}$ for the 98 finger transistor

\section{CONCLUSions}

We have demonstrated a practical approach for developing geometrically scalable thermal models that can be used during the design process of high power RF transistors. The model is expressed as a thermal impedance matrix whose element values are calculated using finite-element thermal flow simulations and thin-plate splines. The model is fast to simulate while retaining the underlying accuracy of the finite-element simulation. We have demonstrated the scalability of the model by calculating the temperature distribution over a 98 -finger

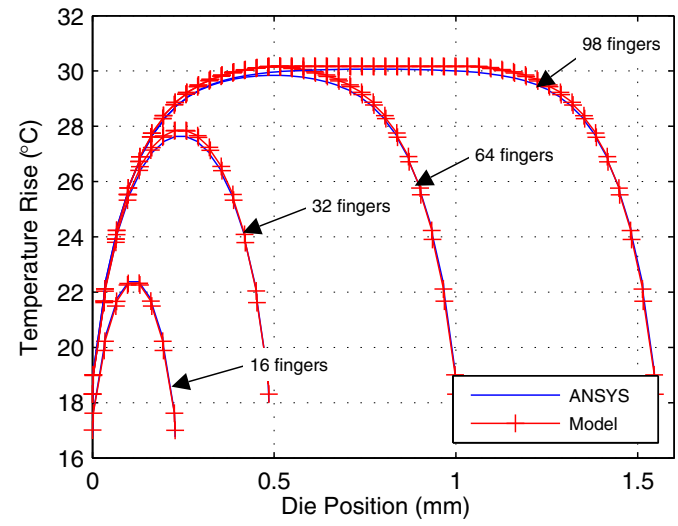

Figure 6. Scalability plot showing all of the 16, 32, 64, 98 fingers.

device that was determined from simulation of a 32-finger base structure.

\section{ACKNOWLEDGMENTS}

The authors would like to acknowledge Michael Guyonnet for his thoughtful comments and insights on thermal model development, and David Burdeaux for the illustration and photograph of the transistor.

\section{REFERENCES}

[1] S. Wunsche, C. Clauss, P. Schwarz, and F. Wikler, "Electro-thermal circuit simulation using simulator coupling," IEEE Trans. VLSI Syst., vol. 5, no. 3, pp. 277-282, Sep. 1997.

[2] ANSYS Inc., Canonsburg, PA, U.S.A

[3] Comsol Multiphysics, Comsol Inc., Los Angeles, CA, U.S.A.

[4] W. Batty, C. E. Christoffersen, A. J. Panks, S. David, C. M. Snowden, and M. B. Steer, "Electrothermal CAD of power devices and circuits with fully physical time-dependent compact thermal modeling of complex non-linear 3-D systems," IEEE Trans. Adv. Packag., vol. 24, no. 4, pp. 566-590, Dec. 2001.

[5] R. Sommet, D. Lopez, and R. Quere, "From 3D thermal simulation of HBT devices to their thermal model integration into circuit simulators via Ritz vectors reduction technique," in 8th ITHERM., May 2002, pp. $22-28$

[6] D. Dennis, C. M. Snowden, and I. C. Hunter, "Coupled electrothermal, electromagnetic, and physical modeling of microwave power FETs," IEEE Trans. Microw. Theory Tech., vol. 54, no. 6, pp. 2465-2470, Jun. 2006.

[7] J. Wood, P. H. Aaen, D. Bridges, D. Lamey, M. Guyonnet, D. S. Chan, and N. Monsauret, "A nonlinear electro-thermal scalable model for highpower LDMOS transistors," IEEE Trans. Microw. Theory Tech., vol. 57, no. 2, pp. 282-292, Feb. 2009.

[8] M. Mahalingam and E. Mares, "Infrared temperature characterization of high power RF devices," in IEEE MTT-S Int. Microwave Symp. Dig., vol. 3, May 2001, pp. 2199-2202.

[9] P. H. Aaen, J. A. Plá, and J. Wood, Modeling and Characterization of RF and Microwave Power FETs. Cambridge, UK: Cambridge University Press, 2007.

[10] M. Mahalingam and E. Mares, "Thermal measurement methodology of RF power amplifiers," 2004, freescale Semiconductor, Inc. [Online]. Available: http://www.freescale.com

[11] J. Sofia, "Fundamentals of thermal resistance measurement," 1995, analysis Tech., Inc. [Online]. Available: www.analysistech.com/ downloads/fundamen.pdf

[12] Matlab Spline Toolbox v3.2.2 User Manual, Mathworks, Inc.

[13] M. Guyonnet, R. Sommet, R. Quere, and G. Bouisse, "Non-linear electro thermal model of LDMOS power transistor coupled to 3D thermal model in a circuit simulator," in Proc. BCTM, Sep. 2003, pp. 11-14. 